\title{
Greatly improved sperm motility from vas deferens sperm retrieval: a case for accessory gland related subfertility in spinal cord injured man. Case report
}

\author{
J P Buch MD
}

Division of Urology and Surgical Research Center, Department of Surgery, University of Connecticut Health Center, Farmington, Connecticut 06030-3955, USA.

\begin{abstract}
When sperm motility from vibratory or electroejaculates of spinal cord injured men reveals consistently poor motility $(<10 \%)$, one should consider accessory gland factors as a potential cause. Herein, we report the case of a 22 year old paraplegic male who consistently had motility of $1-2 \%$ on electroejaculates. He was evaluated in the office with vas deferens sperm retrieval, which revealed a $0.4 \mathrm{ml}$ volume, 9 million $/ \mathrm{ml}$ density, and $67 \%$ motility. We suggest that this significant percent motility differential can only be explained by an accessory gland factor impacting negatively upon this individual's fertility. We propose vas deferens sperm retrieval as a potentially diagnostic and therapeutic procedure in similar cases.
\end{abstract}

Keywords: spermatozoa; spinal cord injury; vas deferens; male infertility.

\section{Introduction}

Consistent defects have been noted in ejaculates obtained from spinal cord injured men who have failure of normal ability to ejaculate after injury. A variety of techniques, including external vibratory massage, rectal probe electroejaculation, and vas deferens sperm retrieval have been used to obtain sperm for insemination of female partners of spinal cord injured men with failure to ejaculate spontaneously. ${ }^{1-9}$ In the great majority of such men, the sperm numbers appear adequate; however, there is a consistent defect of motility in which the percent of sperm mobility typically averages only $20 \% .^{4,6,7}$ In the normal fertile population of non spinal cord injured men, the percent mobility should be at least $60 \%$ or greater. ${ }^{10} \mathrm{~A}$ number of theories exist to explain the defects in sperm motility, sperm survival in cold storage, and ability of sperm to fertilize eggs that are found in spinal cord injured men. Such potential etiological factors include recurrent urinary tract infections, neurogenic effects of the spinal injury, subclinical endocrine changes related to the neurological injury, and temperature or pressure related effects of the typical closed- leg position. ${ }^{7}$ Herein, we report the case of a spinal cord injured man who, on repeated electroejaculation specimens, was noted to have poor motility and very poor sperm survival in either sperm processing or in attempts at sperm penetration assay. In a single episode used for insemination, significantly improved motility was noted in a specimen obtained directly from the vas deferens, which maintained an excellent motile yield on sperm processing. This case provides strong circumstantial evidence for the existence of an accessory gland related male fertility factor in at least some spinal cord injured men, which can be circumvented by vas deferens sperm retrieval.

\section{Case report}

At the time of initial presentation, the patient, a 22 year old Hispanic man with T12 incomplete paraplegia of 5 years' duration secondary to a gunshot wound, had been able to have intercourse with satisfactory erection without medical assistance, but unfortunately had been unable to reach climax and ejaculate, even with attempts at vibratory stimulation. He managed his neuropathic bladder with intermittent self catheterization. He had a seizure disorder, for 
which he took Dilantin (400 mg daily). On clinical examination he had some sensation over the anterior medial thighs and scrotum, as well as some hip abduction and some hip adduction, as well as minimal flexion of the left hip. He had a normal circumcised phallus and normal bilaterally descended testicles of normal size, with no evdience of epididymal or vas deferens pathology. His bulbocavernous reflex was intact, but diminished.

Attempts at vibratory stimulation in the office failed to achieve ejaculation. On initial attempt at rectal probe electroejaculation, due to his incomplete lesion, we were able to achieve a maximum of only 11 volts stimulation before abdominal cramping and left leg pain resulted in termination of the procedure. His maximum erection at that time was $50 \%$. This resulted in both antegrade and retrograde ejaculation. He had $1 \mathrm{ml}$ antegrade ejaculation, with 25 million per $\mathrm{ml}$ sperm density, and only $1 \%$ poorly progressive motility. The retrograde specimen, however, was centrifuged down to a volume of $2 \mathrm{ml}$, with a final density of 175 million per $\mathrm{ml}$, with $15 \%$ motility and grade 2 on a scale of 0 to 4 of forward progression. This specimen was tested for sperm antibodies, for which it was found to be negative using the immunobead test. The specimen was also cryopreserved according to a standard protocol, ${ }^{7}$ and thawed with only $1 \%$ motility.

During the next session 2 weeks later, again, antegrade and retrograde ejaculates were obtained. Antegrade had $1 \mathrm{ml}$ volume, with 27 million per ml density and only $1 \%$ motility. Forty-eight million round cells per $\mathrm{ml}$ were noted. In the retrograde, the final volume was $4 \mathrm{ml}$ after centrifugation, with 183 million per $\mathrm{ml}$ density, $15 \%$ motility, with a grade of 2.5 . Round cell density was 14 million per ml. This was tested in the optimized sperm penetration assay according to published protocols. ${ }^{7.11} \mathrm{He}$ penetrated only one of seven eggs, with a penetration rate of 0.14 (normal penetration rate being 5 or greater). On that basis, he manifested significantly decreased fertility potential into the low subfertile/near sterile range. However, since his fiancee had two children from a prior relationship, and since he had demonstrated at least some sperm ability to penetrate eggs, we proceeded with two subsequent cycles of intrauterine insemination using electroejaculated specimens. On these next two cycles, appropriate sperm densities of 150 million were obtained. However, motility was low, at only $1-2 \%$. He had had no evidence of clinical urinary infections over this entire interval.
On the basis of a suspected accessory gland factor, and due to the fact that on the second cycle his initial motility was $1 \%$, with no motility after sperm processing, we decided to attempt a vas deferens sperm retrieval. This was performed in the office under local anesthesia, using a vasotomy technique as previously reported. ${ }^{89}$ Exploration of the right side revealed no significant fluid being milked forth. This incision was closed after loupe $(4.5 \times)$ magnification closure of the vasotomy. Vasotomy on the second side was performed, revealing small amounts of fluid. On both sides, the distal portion of the vas irrigated easily with $3 \mathrm{cc}$ saline, ruling out any ejaculatory duct obstruction. A specimen was obtained, which revealed $0.4 \mathrm{ml}$ volume, 9 million per $\mathrm{ml}$ density, with $67 \%$ progressive motility and $41 \%$ normal morphology, with no evidence of round cells. This specimen was prepared by standard sperm processing with discontinuous mini-Percoll density gradient centrifugation modified after Ord et al. ${ }^{12}$ It was resuspended back to a density of 7 million per $\mathrm{ml}$, with $72 \%$ motility in a $0.5 \mathrm{ml}$ volume for intrauterine insemination. Therefore, the total number of motile sperm available for insemination was approximately 2.5 million. This was an approximately $100 \%$ recovery of all the motile sperm that were present before processing. Although a pregnancy was not achieved from this cycle, and this couple has undergone no further insemination cycles, it was our plan to consider doing further cycles with this protocol, with more careful stimulation and timing of the ovulation.

\section{Discussion}

Previously, there have been two reports of using vas deferens sperm retrieval in cases of neurogenic failure of ejaculation. ${ }^{8.9}$ In neither of these two reports was the result of vas deferens sperm retrieval compared with what was available from either vibratory ejaculation or electroejaculation. This case represents clinical evidence that in at least some spinal cord injured men, greatly superior sperm specimens may be obtained from the vas deferens than are obtained from the ejaculated specimens. Presumably, the reason for this would be a defect at the level of the accessory sex glands in terms of the quality and character of the fluid which is added to the sperm at that level. The basis for this statement is the fact that there was no significant resistance to vasography to 
incriminate any ejaculatory duct obstruction in this patient. The potential etiology for decreased quality of these ejaculates could be either neurogenic-related decrease in quality of the secretions, or infectionrelated, or both. The possible association of these factors with the presence of white blood cells in the ejaculate was an inconsistent finding. In one antegrade ejaculate with low motility, there was a very high round cell density of 48 million per ml; however, in the most recent electroejaculation specimen with only $1 \%$ motility, there were only 4 million round cells per $\mathrm{ml}$. The exact deficiencies in the seminal plasma in these cases will await further study and definition. In a prior study of cryopreservation protocols for fertile and infertile sperm, we have also noted evidence for accessory gland (seminal fluid) related fertility factors. ${ }^{13}$

Support for the theory of accessory gland inflammation resulting in poor sperm function which can be circumvented by vas deferens or epididymis sperm retrieval has recently been provided in a report by Tanaka. ${ }^{14}$ In that study, sperm with enhanced motility were retrieved from the epididymis of several (non spinal injured) men with poor motility presumed secondary to accessory gland inflammation beyond the epididymis. This procedure was successfully combined with in vitro fertilization (IVF) in some couples with previously failed IVF cycles attributed to low sperm motility $(<20 \%)$.

The potential for vas deferens sperm to achieve pregnancies when combined with intrauterine insemination has already been well documented in at least two reports. ${ }^{8,9}$ Furthermore, the potential for cryopreservation of vas deferens sperm for use in subsequent insemination cycles has also been recently reported. ${ }^{15}$ In this way, it is also possible that the vas deferens sperm retrieval may not have to be timed exactly with the wife's ovulatory cycle if a successful cryopreservation can be performed. Regardless, this approach of vas deferens sperm retrieval combined with intrauterine insemination in the anejaculatory spinal cord injured male presents a viable option for achieving pregnancy.

\section{Conclusions and recommendations}

When faced with a spinal cord injured man who has failure to ejaculate, one should begin with attempts at external vibratory stimulation. According to Brindley, ${ }^{3}$ the highest success rates are predicted from men who demonstrate hip flexion with stimulation of the sole of the foot. In such cases, the success rate of external vibratory stimulation may exceed $50 \%$. Should external vibratory stimulation fail, rectal probe electroejaculation provides an excellent and reliable method for obtaining ejaculates in at least $90 \%$ of such cases. ${ }^{7}$ However, when the motility of the sperm in these specimens is consistently low ( $10 \%$ or less), or the motile sperm yield from sperm processing is consistently pour, one should consider the possibility of vas deferens exploration and sperm retrieval as a treatment for the potential of an accessory gland-related fertility disorder. This does not necessarily need to be performed at the time of insemination, and on a first-evaluation basis cryopreservation could also be performed. These findings and recommendations will require further corroboration by evaluation of future patients.

\section{Acknowledgement}

This study was supported in part by a grant from the Spinal Cord Research Foundation of the Paralyzed Veterans of America.

\section{References}

1 Horne HW, Paull DP, Munro D (1948) Fertility studies in the human male with traumatic injuries of the spinal cord and cauda equina. $N$ Engl J Med 239: 959-961.

2 Brindley GS (1981) Electroejaculation: its technique, neurological implications and uses. J Neurol Neurosurg Psychiatry 44: 9-18.

3 Brindley GS (1984) The fertility of men with spinal injuries. Paraplegia 22: 337-348.

4 Perkash I, Martin DE, Warner $\mathrm{H}$ et al (1985) Reproductive biology of paraplegics: results of semen collection, testicular biopsy and serum hormone evaluation. J Urol 134: 284-288. 
5 Sarkarati M, Rossier A, Fam BA (1987) Experience in vibratory and electroejaculation techniques in spinal cord injury patients: a preliminary report. J Urol 138: 59-62.

6 Bennett CJ, Seager SW, Vasher EA, McGuire EJ (1988) Sexual dysfunction and electroejaculation in men with spinal cord injury: review. J Urol 139: 453-457.

7 Buch JP, Zorn BH (1993) Evaluation and treatment of infertility in spinal cord injured men through rectal probe electroejaculation. J Urol 149: 1350-1354.

8 Berger RE, Muller CH, Smith D et al (1986) Operative recovery of vasal sperm from anejaculatory men: preliminary report. J Urol 135: 948-950.

9 Bustillo M, Rajfer J (1986) Pregnancy following insemination with sperm aspirated directly from vas deferens. Fertil Steril 46: 144-146.

10 Lipshultz LI, Howards SS, Buch JP (1991) Male infertility. In: Gillenwater JY, Grayhack J, Howards S, Duckett J, editors. Adult and Pediatric Urology. 2nd ed. Mosby Yearbook, Chicago: 1425-1477.

11 Johnson A, Bassham B, Lipshultz LI, Lamb DJ (1990) Methodology for the Optimized Sperm Penetration Assay. In: Keel BA, Webster BW, editors. Handbook of the Laboratory Diagnosis and Treatment of Infertility. CRC Press, Boca Raton, FL: 135-147.

12 Ord T, Patrizio P, Marello E et al (1990) Mini-Percoll: a new method of semen preparation for IVF in severe male factor infertility. Hum Reprod 5: 987-989.

13 Kolon TF, Phillips KA, Buch JP (1992) Custom cryopreservation of human semen. Fertil Steril 58: $1020-1023$.

14 Tanaka A (1992) A new sperm collection method for in vitro fertilization: collection at tail of epididymis. Hum Reprod 7: 838-840.

15 Buch JP, Phillips KA, Kolon TF (1992) Cryopreservation of microscopically extracted ductal sperm: pentoxifylline enhancement of motility (abstract 0-076). Presented at the 48th Annual Meeting of the American Fertility Society, New Orleans, Louisiana, November 2-5, 1992. 